\title{
C-Mode Real Time Tomographic Reflection for a Matrix Array Ultrasound Sonic Flashlight
}

\author{
George Stetten ${ }^{1,2,3}$, Aaron Cois ${ }^{1,2}$, Wilson Chang ${ }^{1,2,3}$, Damion Shelton ${ }^{2}$, \\ Robert Tamburo ${ }^{1,2}$, John Castellucci ${ }^{4}$, and Olaf von Ramm ${ }^{4}$ \\ ${ }^{1}$ Department of Bioengineering, University of Pittsburgh, \\ ${ }^{2}$ Robotics Institute, Carnegie Mellon University, \\ ${ }^{3}$ University of Pittsburgh Medical Center \\ ${ }^{4}$ Department of Biomedical Engineering, Duke University \\ http://www.vialab.org
}

\begin{abstract}
Real Time Tomographic Reflection (RTTR), permits in situ visualization of tomographic images, so that natural hand-eye coordination can be employed directly during invasive procedures. The method merges the visual outer surface of the patient with a simultaneous scan of the patient's interior, using a half-silvered mirror. A viewpoint-independent virtual image is reflected precisely into the proper location. When applied to ultrasound, we call the resulting RTTR device the sonic flashlight. We have previously implemented the sonic flashlight using conventional 2D ultrasound. In this paper we present the first images from a new sonic flashlight based on Real Time 3D (RT3D) ultrasound, which uses a matrix array to electronically steer the ultrasound beam at very high speed. We show in situ C-mode images, which are parallel to the face of the transducer, of the hand and the cardiac ventricles.
\end{abstract}

\section{Introduction}

In the practice of medicine, the standard method of viewing an image is to examine a film or screen, rather than to look directly into the patient. A number of researchers have worked to develop more natural ways to merge images with the perceptual real world. [1-4] We have previously reported the concept of Real Time Tomographic Reflection (RTTR), and applied it successfully to ultrasound. [5,6] Conventional ultrasound produces a tomographic slice within the patient representing a set of 3D locations that lie in a plane. The image of that tomographic slice, displayed on a flat panel monitor at the correct size, may be reflected to occupy the same physical space as the actual slice within the patient. If a half-silvered mirror is used, the patient may be viewed through the mirror with the reflected image of the slice accurately superimposed on the patient, independent of viewer location. The reflected image is truly occupying its correct location and does not require any particular perspective to be rendered correctly.

To accomplish RTTR, certain geometric relationships must exist between the slice being scanned, the monitor displaying the slice, and the mirror. As shown in 


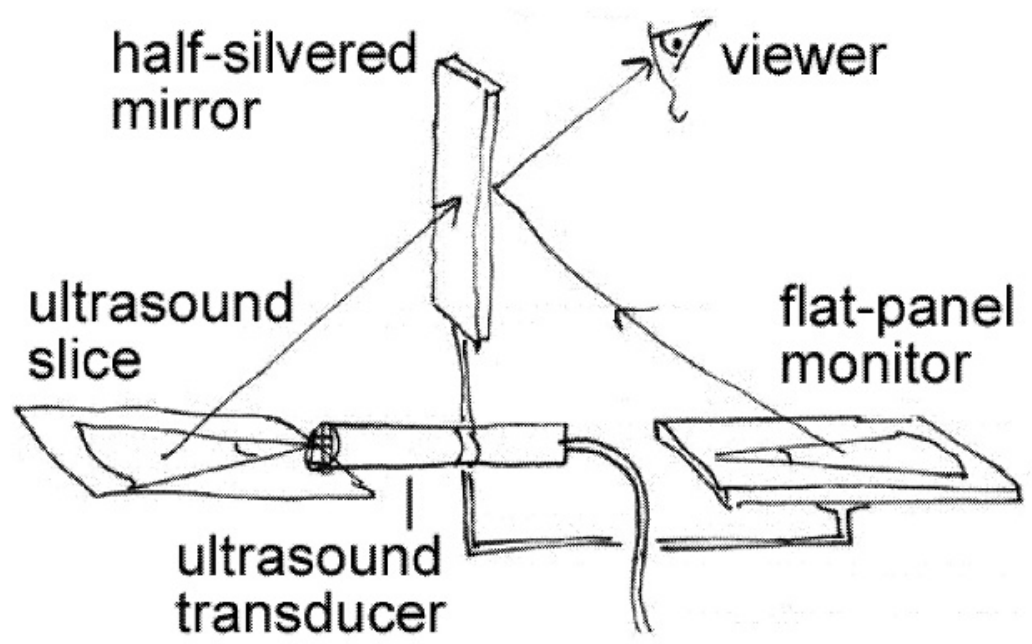

Fig. 1. Schematic representation of an ultrasound RTTR device, or sonic flashlight. A flatpanel monitor and an ultrasound transducer are placed on opposite sides of a half-silvered mirror to create an in situ virtual image.

Figure 1, the mirror must bisect the angle between the slice and the monitor. On the monitor, the image must be correctly translated and rotated so that each point in the image is paired with its corresponding point in the slice. By fundamental laws of optics, the ultrasound image will appear at its physical location, independent of viewer position.

The RTTR ultrasound apparatus can be constructed in a number of configurations. Figure 2 shows a recent version of a B-mode sonic flashlight, whose images come from a standard ultrasound machine producing a conventional scan along the axis of the ultrasound transducer (Bmode image). Thus, the flat panel monitor is mounted as shown, also along the axis of the transducer with the mirror bisecting the angle between the transducer and the flat-panel monitor. The

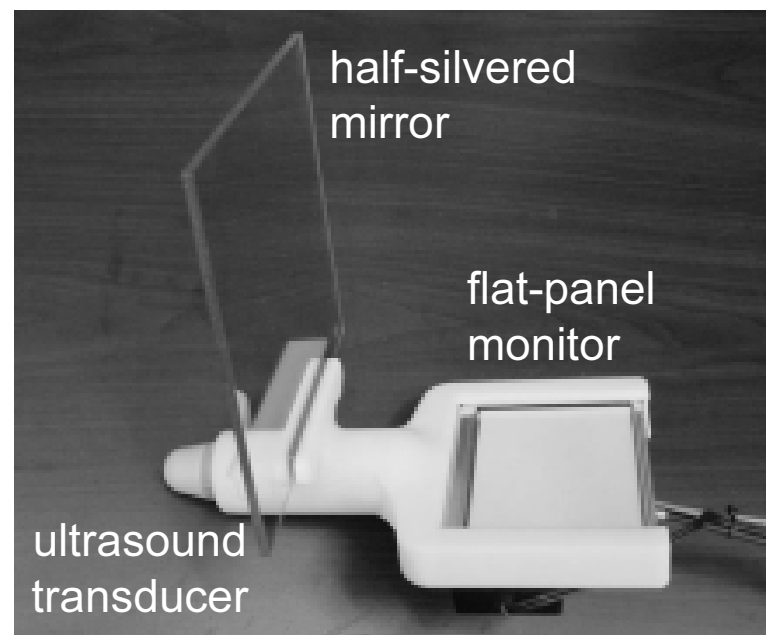

Fig. 2. Recent prototype of the sonic flashlight, showing the relationship of its components. mirror is mounted perpendicular to the axis, so that the image on the flat panel monitor is reflected to occupy the space being 


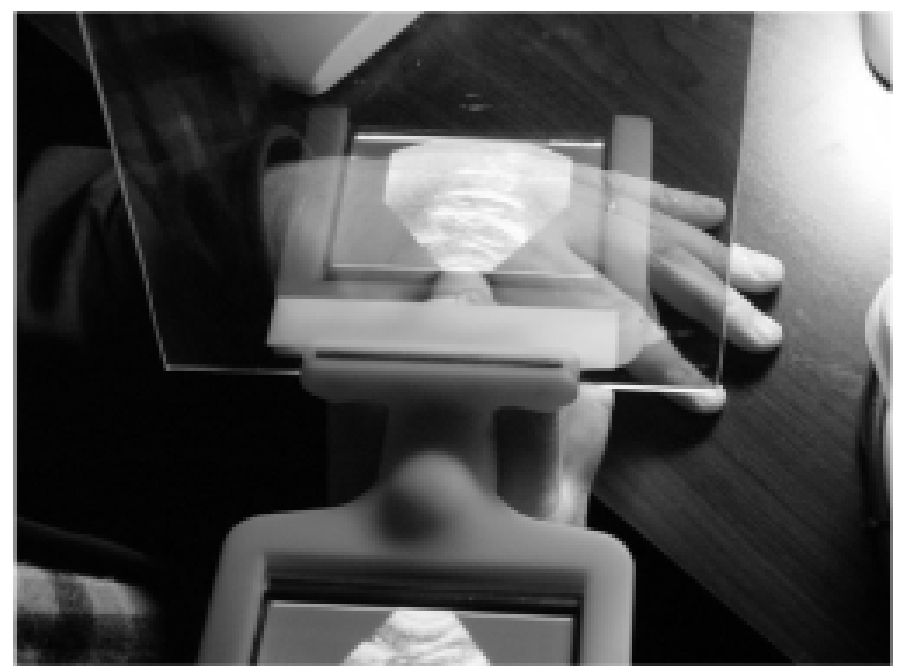

Fig. 3. Photograph from the viewpoint of the operator, showing a scan of the hand using the apparatus in Figure 2. The reflected ultrasound image is merged with the direct visual image.

scanned by the ultrasound transducer. The device is designed to be held in one hand while performing an invasive procedure with the other.

In Figure 3, the same sonic flashlight from Figure 2 is shown in use. A human hand is seen with the transducer pressed against the soft tissue between the thumb and index finger. While not a common target for clinical ultrasound, the hand was chosen because it clearly demonstrates successful alignment. The external surfaces of the hand are located consistent with structures within the ultrasound image. The photograph cannot convey the strong sense, derived from stereoscopic vision, that the reflected image is located within the hand. This sense is intensified by head motion because the image remains properly aligned from different viewpoints. To one experiencing the technique in person, ultrasound targets within the hand would clearly be accessible to direct percutaneous injection, biopsy or excision.

\section{Real Time 3D Ultrasound}

This paper presents the first application (as far as we know) of RTTR to 3D ultrasound. In particular, we have chosen Real Time 3D (RT3D) ultrasound, which has been available commercially since the mid 1990's through Volumetrics Medical Imaging, Inc., a spin-off from Duke University, where we participated in development of the technology. [7,8] Volumetrics sold fewer than 25 scanners in the United States and abroad. The particular scanner that we used for the C-mode sonic flashlight was known as "T4", the prototype for the commercialized scanners. Phillips, Inc., has recently introduced a similar RT3D scanner commercially. Previously, all other commercial 3D ultrasound scanners gathered 3D data by mechanically "wobbling" a 
conventional array through the third dimension. Since the matrix-array (Figure 4) of the RT3D scanner, by contrast, has no moving parts, it can achieve rapid scan rates (22 entire volumes per second), permitting the construction of a sonic flashlight with no apparent latency. Although developed originally for cardiac applications, where high speed is essential, the same speed is also well suited to real-time guidance of invasive procedures.
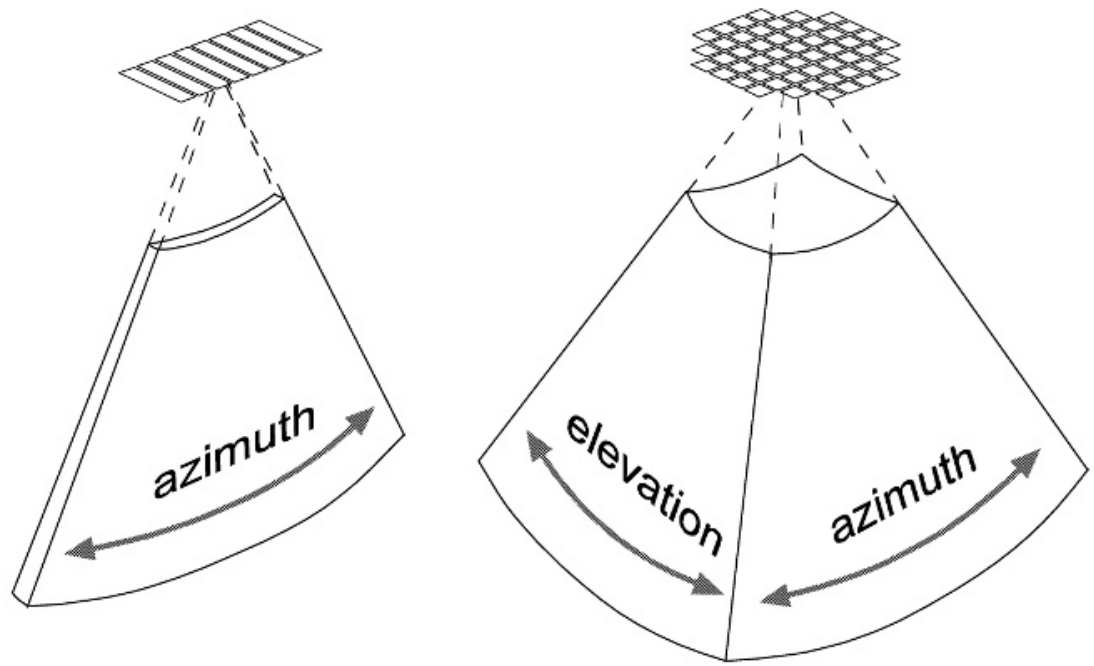

Fig. 4. The conventional $2 \mathrm{D}$ ultrasound scanner uses a row of transducer elements to steer the direction of transmission and reception along the azimuth dimension, capturing a single sector or B-mode slice (left). The Real Time 3D Ultrasound (RT3D) scanner uses a matrix-array to steer in both azimuth and elevation, capturing an entire round-bottomed "pyramid" of data (right).

Given the high-speed volumetric data from the Duke RT3D ultrasound machine, we can generate in situ slices with any orientation relative to the transducer For example, the scanner permits two orthogonal B-mode slices to be visualized simultaneously, as well as a so-called "C-mode" slice, parallel to the face of the transducer at any depth within the pyramid.

\section{C-Mode Version of the Sonic Flashlight}

A diagram of our C-mode sonic flashlight is shown in Figure 5 and the actual apparatus is shown in Figure 6. Unlike the B-mode sonic flashlight in Figures 1-3, the flat panel monitor in the $\mathrm{C}$-mode sonic flashlight is mounted perpendicular to the ultrasound probe, so that its reflection occupies a C-mode slice on the other side of the mirror. The monitor is mounted in a track to permit selection of the appropriate depth for any C-mode slice within the 3D ultrasound scan. 


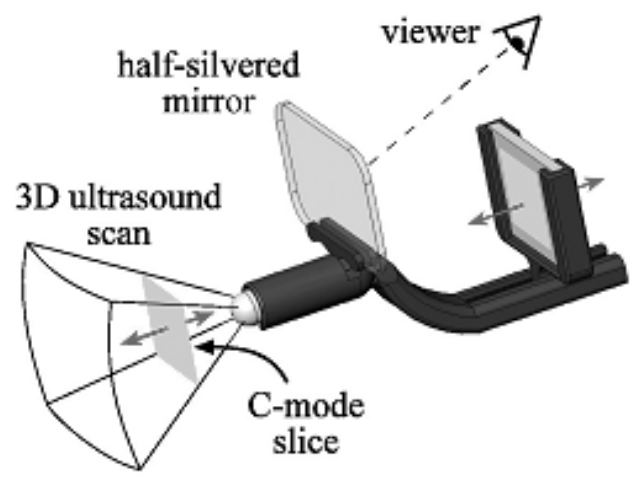

Fig. 5. Diagram of C-mode sonic flashlight based on Real Time 3D ultrasound.

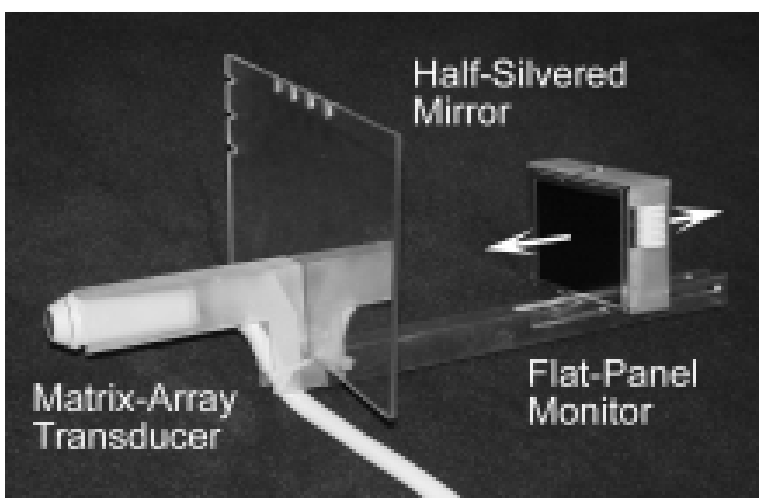

Fig. 6. Implementation of C-mode sonic flashlight. Flat-panel monitor can slide in track to adjust the virtual image to the appropriate depth.

\section{Calibration}

Clearly, an accurate method for calibration is required. Without it, the sonic flashlight would be unable to guide an invasive procedure safely. Calibration requires careful consideration of the degrees of freedom in the registration process. The challenge is to make each pixel in the virtual image occupy, and therefore

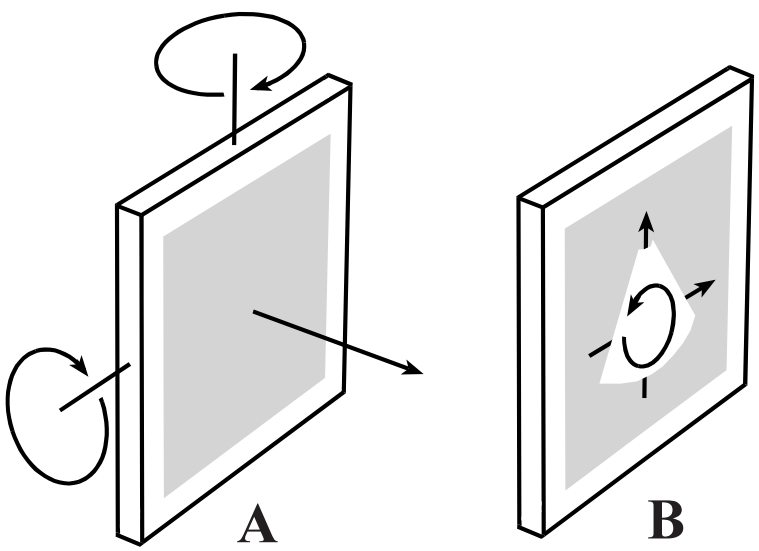

Fig. 7. Transforms for (A) physically moving the display, and (B) moving the image on the screen. 
appear to emanate from, its actual 3D location in the slice. For the sake of this experiment we consider only the geometric transform of a rigid body, i.e., we assume that the ultrasound slice is displayed without distortion at its correct scale on a perfectly flat monitor (Figure 7). The geometric transform required to superimpose the virtual image onto the slice can be represented as two sets of translations and rotations, each of which has 3 degrees of freedom. The first ( 2 rotations and 1 translation) allows the flat panel display to be moved into its correct plane, making the virtual image coplanar with the actual ultrasound slice. We can achieve this by physically moving the display in its track, and by ensuring that the display and the mirror are both parallel to the face of the ultrasound transducer. The second (two translations and one rotation) are achieved by adjusting the image of the ultrasound slice on the flat panel monitor.

For the initial prototype of the C-mode Sonic Flashlight, calibration was achieved by adjusting the depth of the virtual image to the known depth of the slice as determined by the scanner. Center location and aspect ratio (1:1) of the image on the screen was adjusted by eye. Scale was then adjusted visually, to produce matching motion between the ultrasound image and surface landmarks as the transducer was passed over the target.

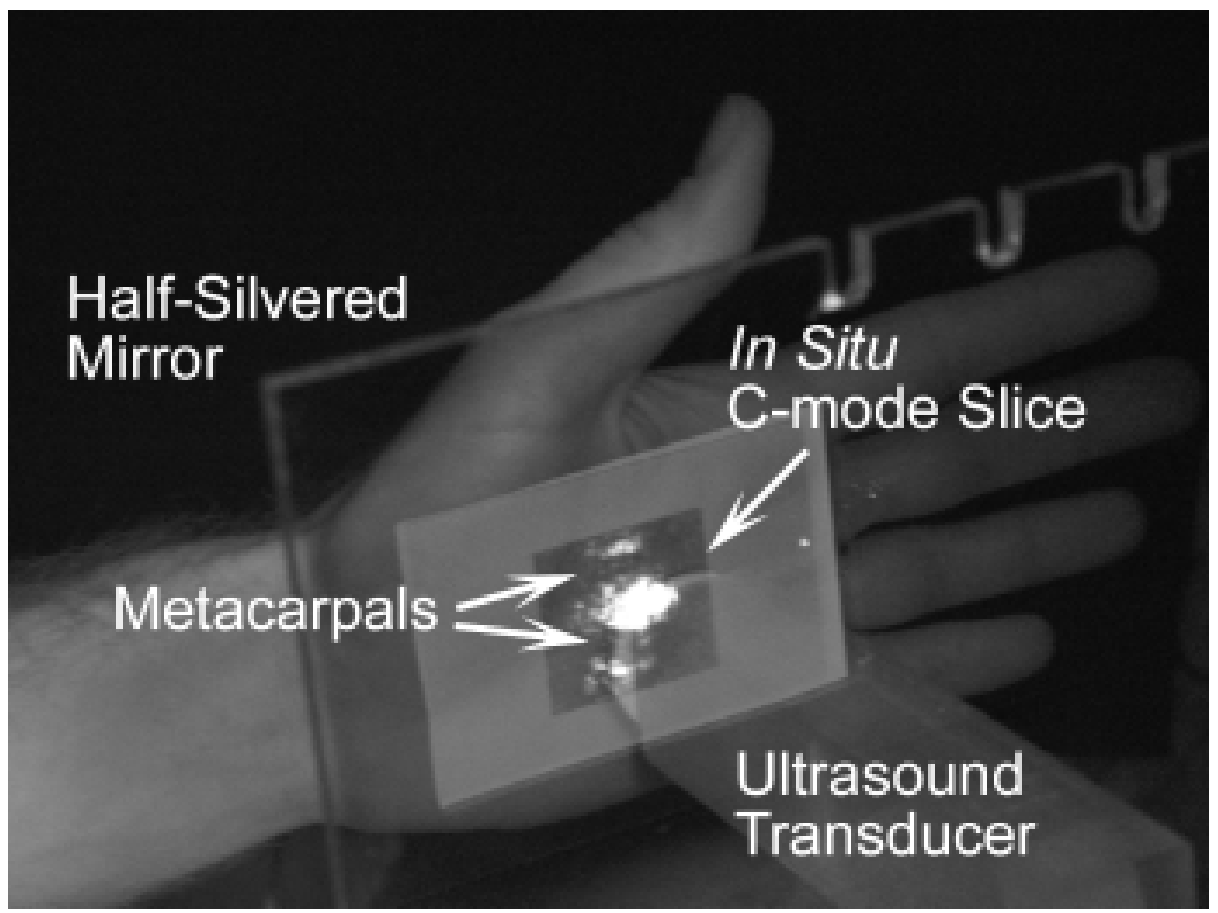

Fig. 8. The C-Mode sonic flashlight produces an in situ image of the hand, showing the $3^{\text {rd }}$ and $4^{\text {th }}$ metacarpals at their appropriate locations. 


\section{Results}

Figure 8 shows a $\mathrm{C}$-mode image of the bones in the hand. The $\mathrm{C}$-mode image appears more naturally "illuminated" by the ultrasound than a B-mode image, because the Cmode slice cuts across the ultrasound beam and its pixels do not block each other along the ultrasound beam. The C-mode image therefore does not include the shadows that normally streak across a B-mode image. Structures in the C-mode image appear to reflect ultrasound the way they would reflect light. Whereas the Bmode Sonic Flashlight "looks" like a flashlight, the C-mode Sonic Flashlight "acts" like a flashlight. Figure 9 shows the left ventricle (LV) and right ventricle (RV) of a human heart in their appropriate locations.

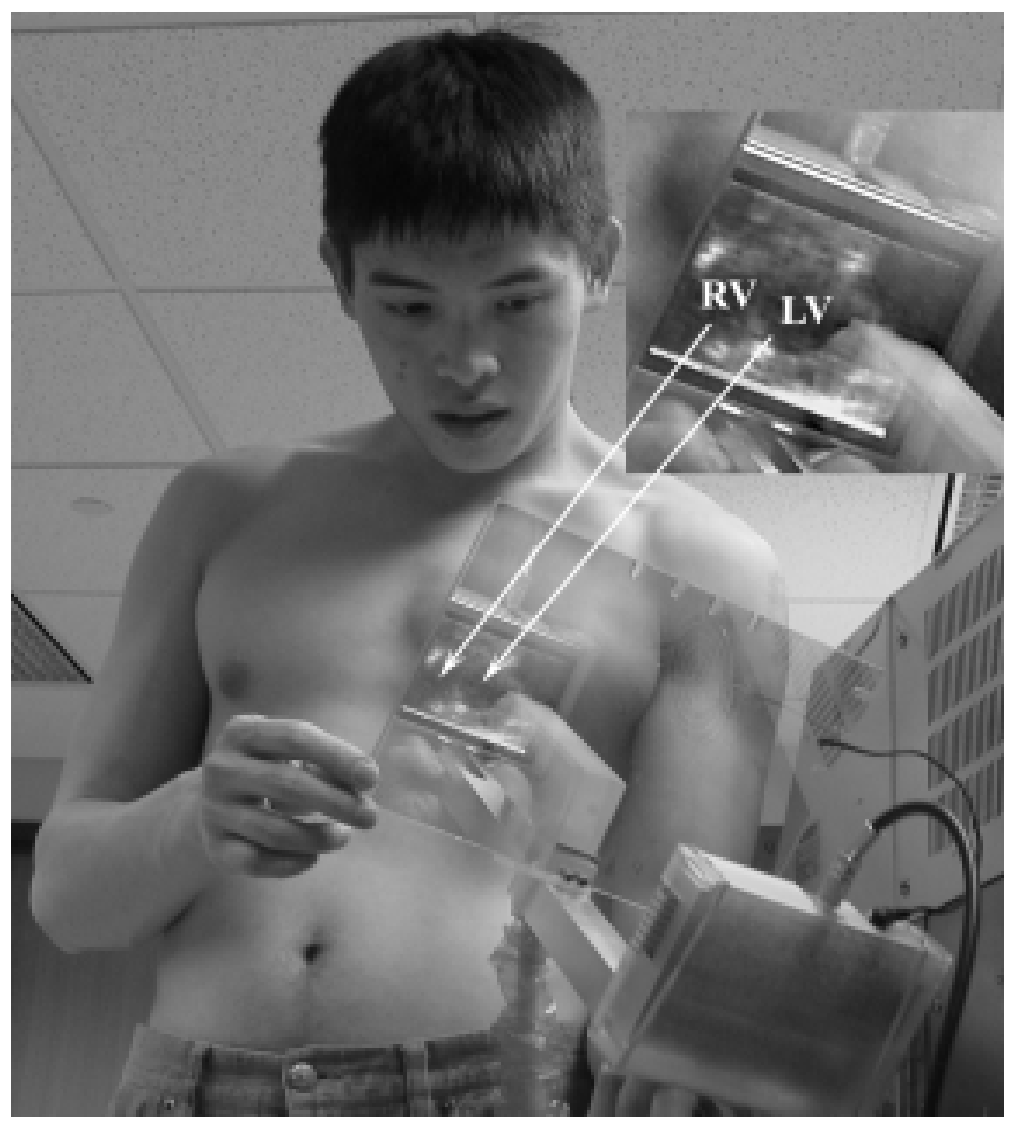

Fig. 9. Cardiac ventricles ( $L V=$ left ventricle, $R V=$ right ventricle) seen in situ using the $C$ mode sonic flashlight. Insert is magnified to show the correct shape of each ventricle. The LV is round, while the RV is crescent-shaped. 


\section{Discussion}

Superimposing ultrasound images on human vision using RTTR may improve an operator's ability to find targets while avoiding damage to neighboring structures and facilitating interpretation of ultrasound images by relating them spatially to external anatomy. As such, it holds promise for increasing accuracy, ease, and safety during percutaneous biopsy of suspected tumors, amniocentesis, fetal surgery, brain surgery, insertion of catheters, and many other interventional procedures. The extension of RTTR to matrix array Real-Time 3D ultrasound offers the ability to visualize in situ slices other than the conventional B-mode, including C-mode slices parallel to the face of the transducer. This may be advantageous in particular invasive procedures by allowing slice orientations with greater accessibility from the body surface.

The sonic flashlight has been awarded a US Patent. [9]

\section{References}

1. State, A., Livingston, M., Garret, W., Hirota, G., Whitton, M., Pisano, E. \& Fuchs, H., Technologies for Augmented Reality Systems: Realizing Ultrasound-Guided Needle Biopsies. ACM SIGGRAPH, New Orleans, LA, 439-446 (1996).

2. Rosenthal, M., State, A., Lee, J., Hirota, G., Ackerman, J., Keller, K., Pisano, E., Jiroutek, M., Muller, K. \& Fuchs, H., Augmented Reality Guidance for Needle Biopsies: A Randomized, Controlled Trial in Phantoms. MICCAI 2001, Lecture Notes in Computer Science 2208, Springer-Verlag, 240-248.

3. Sauer, F., Khamene, A., Bascle, B., Schimmang, L., Wenzel, F. \& Vogt, S., Agumetned reality visualization of ultrasound images: System description, calibration, and features. International Symposium on Augmented Reality 2001, IEEE and ACM, New York City, 30-39.

4. Masamune, K., Fichtinger, G., Deguet, A., Matsuka, D. \& Taylor, R., An Image Overlay System with Enhanced Reality for Percutaneous Therapy Performed Inside CT Scanner. MICCAI 2002, Lecture Notes in Computer Science, 2489, Springer-Verlag, 77-84.

5. Stetten, G., Chib, V. \& Tamburo, R., System for Location-Merging Ultrasound Images with Human Vision. Applied Imagery Pattern Recognition (AIPR) 2000, Workshop, IEEE Computer Society, Washington, DC, 200-205.

6. Stetten, G. \& Chib, V., Overlaying ultrasound images on direct vision. Journal of Ultrasound in Medicine 20, 235-240 (2001).

7. von Ramm, O. T., Smith, S. W. \& Pavy, H. G., Jr., High-speed ultrasound volumetric imaging system - Part II: Parallel processing and image display. IEEE Transactions on Ultrasonics, Ferroelectrics, and Frequency Control 38, 109-115 (1991).

8 Stetten, G., Ota, T., Ohazama, C., Fleishman, C., Castelucci, J., Oxaal, J., Ryan, T., Kisslo, J. \& Ramm, O. v., Real-Time 3D Ultrasound: A New Look at the Heart. Journal of Cardiovascular Diagnosis and Procedures 73-84 (1998).

9 Stetten, G. U.S. Patent no. 6,599,247, "System and Method for Location-Merging of RealTime Tomographic Slice Images with Human Vision,” July 29, 2003. 